\title{
Primary prevention of sudden cardiac death: one step forwards and one step backwards
}

\author{
Michael E Cain
}

In 2006, ventricular tachycardia (VT) and ventricular fibrillation (VF) remain a leading cause of mortality worldwide. Judging from data acquired in the US and Europe, VTNF claims two to three lives per 100,000 individuals each year. The incidence of arrhythmic death has not declined in tandem with advances in the management of acute coronary syndromes, heart failure and cardiovascular disease in general. Primary prevention of sudden death is desirable. It requires accurate identification of patients at risk and effective therapies that prevent or terminate VT/VF.

A step forward in care was achieved when results of the Multicenter Unsustained Tachycardia Trial, the Multicenter Automatic Defibrillator Implantation Trials, and the Sudden Cardiac Death in Heart Failure Trial demonstrated a favorable impact of implantable cardioverter-defibrillator (ICD) therapy on mortality and arrhythmic death in patients with severely reduced ventricular function due to ischemic or nonischemic cardiomyopathy. The science of primary prevention, however, took a step backwards because reduced left ventricular ejection fraction was inadvertently established as the optimum surrogate of vulnerability to VT/VF and, thereby, the need for ICD therapy. Prescription of ICDs for primary prevention is currently restricted to patients in this single-risk-factor group. Many individuals with low ejection fractions do not die suddenly. Moreover, the majority of actual arrhythmic deaths do not occur in this subset.

The reliance on ejection fraction also detracts from decades of work focused on developing noninvasive approaches to better detect the electrophysiologic abnormalities that initiate and maintain VTNF in patients with structural heart disease. Death of myocardial cells from ischemia, toxic or infectious agents, or pressure or volume overload leads to scar formation, alterations in ventricular geometry, and electrical and anatomic remodeling. The electrophysiologic alterations induced in response to these

\section{Improving \\ our ability to \\ implant reliable \\ ICDs in the \\ right patient is \\ a worthy goal \\ for 2006.}

ME Cain is an

Advisory Board member of Nature

Clinical Practice

Cardiovascular

Medicine.

\section{Competing interests}

The author declared he has no competing interests.

www.nature.com/clinicalpractice doi:10.1038/ncpcardio0503 conditions might occur transiently or develop during the course of healing from injury and persist. They initiate and maintain VT/VF by re-entry, abnormal automaticity, or both. Features that allow detection of the presence of factors known to trigger VT/VF or abnormalities in ventricular conduction and repolarization that are critical to re-entry include the following: long QRS duration on the electrocardiogram or late potentials on the signal-averaged electrocardiogram, indicating slowed conduction; heterogeneities in repolarization shown by QT interval dispersion on electrocardiogram, or on T-wave alternans; imbalance in autonomic tone, indicated by heart rate variability, heart rate turbulence and baroreceptor sensitivity; transient ischemia on stress testing; and ventricular ectopy on Holter monitoring.

While some methods have not lived up to initial expectations, T-wave alternans, signal-averaged electrocardiography, and heart rate turbulence have been shown convincingly to predict which patients will suffer VT/VF. Either, therefore, a risk stratification index should be developed that encompasses several proarrhythmic factors, or none of the current techniques should be viewed as able to completely detect the fingerprint of the relevant electrical abnormalities. Both conclusions are supported.

Consequences of the current state of affairs are that more ICDs are implanted in patients with low ejection fractions than are needed, and not enough primary preventive measures are offered to high-risk patients with less-impaired ventricular function. We should take advantage of this climate to, at worst, test the performance of the most promising risk-stratification approaches, alone or in combination, to assess whether they permit identification of patients with low ejection fraction who will not benefit from an ICD, and, at best, develop novel techniques to detect vulnerability to VT/VF in other patient groups. Improving our ability to implant reliable ICDs in the right patient is a worthy goal for 2006 . 